\title{
Oral Lectures
}

[MS3 - 03] SAXS studies of protein solutions using high brilliance clean beam and low background camera. Nicoleta Galatanu ${ }^{1}$, Sergio Rodrigues $^{1}$, Pierre Panine ${ }^{1}$, Manual Fernandez ${ }^{1}$, Frederic Bossan ${ }^{1}$, Peter Hoghoj ${ }^{1}$, Blandine Lantz $^{1}$, Ronan Mahe ${ }^{1}$

${ }^{1}$ Xenocs, France

Small angle $\mathrm{x}$-ray scattering is a valuable characterization method to study the $3 \mathrm{D}$ envelope of proteins or the arrangement of individual domains in protein complexes providing complementary information to X-ray crystallography or NMR studies. As the measurements are performed in solution it can also be used to provide an insight of sample quality to assess protein targets.

Successful developments of applications in synchrotron beamlines have led to an increase need for high quality laboratory equipments. We will present the latest features and results with the Xeuss SAXS/WAXS equipment from Xenocs on low concentration protein solutions. A new generation of scatterless collimation with variable resolution opens the door to unprecedented ratios of primary beam intensity to background and to measurements on weakly scattering samples.

Short measuring times are accessible through the combination of a low maintenance, high brilliance source with advanced photon counting detectors. This capability will be illustrated with fast sample quality studies depending on concentration (including in situ variation). We will also emphasize the importance of having a low background set-up in particular for measurements at High Q. Combination of SAXS and WAXS measurements for sample investigation will also be discussed. Finally, we will show results of protein size characterization using classical model extrapolation, but also inverse fourier transformation fitting or structure modeling with advanced open source softwares. 